\title{
Application of a novel Anonymization Method for Electrocardiogram data
}

\author{
Zineb Bennis \\ Octopize Mimethic Data, INSA Toulouse \\ zinebennisa@gmail.com
}

\begin{abstract}
Chronos, a time series oriented anonymization method is presented in this paper. The method is tested on electrocardiogram time series in the MIT-BIH Arrhythmia Data Base and the Physikalisch TechnischeBundesanstalt (PTB) Diagnostic ECG, with empirical measures of the privacy protection provided by the method. The signal retention properties of the method are also tested graphically and computationally by training algorithms on the original and anonymized sets. The models trained on the anonymized data achieve a $3 \%$ accuracy difference compared to the original models. The method outputs samples that are similar to the original data while protecting the records by producing new synthetic data points.
\end{abstract}

\section{CCS CONCEPTS}

- time series; • anonymization; • ECG; • ;

\section{KEYWORDS}

synthetic data, data privacy, signals

\section{ACM Reference Format:}

Zineb Bennis and Pierre-Antoine Gourraud. 2021. Application of a novel Anonymization Method for Electrocardiogram data. In ArabWIC 2021: The 7th Annual International Conference on Arab Women in Computing in Conjunction with the 2nd Forum of Women in Research, Sharjah, UAE (ArabWIC 2021), August 25, 26, 2021, Sharjah, United Arab Emirates. ACM, New York, NY, USA, 5 pages. https://doi.org/10.1145/3485557.3485581

\section{INTRODUCTION}

Data privacy has become a leading concern in a more connected world, where data is constantly being generated by all the connected devices around us. New concepts of data as an asset have emerged, companies buy, sell, and extract valuable information every day, and that is especially true in the health domain. Real world data is also extremely important for the assessment of the effectiveness and interactions of new drugs or the development of large-scale scientific studies for example. While these reasons are valid, these endeavors must not come at the expense of the individuals' privacy, whose data can be used without knowledge or consent. That is why international data regulation policies such as the European Data

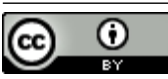

This work is licensed under a Creative Commons Attribution International 4.0 License.

ArabWIC 2021, August 25, 26, 2021, Sharjah, United Arab Emirates

(c) 2021 Copyright held by the owner/author(s)

ACM ISBN 978-1-4503-8418-6/21/08.

https://doi.org/10.1145/3485557.3485581

\author{
Pierre-Antoine Gourraud \\ Université de Nantes, INSERM, CHU de Nantes - Clinique \\ des données \\ pierre-antoine.gourraud@univ-nantes.fr
}

Protection Board (EDPB) or the California Consumer Privacy Act (CCPA) in the United States exist, and why it is necessary to review how data is shared among researchers and companies.

To allow for easy data sharing while protecting the individuals and keeping the statistical value of the data, the scientific community has come up with anonymization methods [8]. These methods take personal data and change it so that the individuals in the data sets are no longer identifiable. While anonymization methods come as a good answer to the concerns mentioned earlier, it is worth noting that their efficiency rests on how well they can retain the valuable information in the data while reducing risks of re-identification. Earlier and simpler anonymization methods rely on data obfuscation and swapping of the features while more sophisticated models may use machine learning and neural networks [11] to achieve this task.

Time series are a specific yet important category of data in that they are of particular interest, especially in the health sector, for medical and pharmaceutical research. This data, in the form of time series records, defines the evolution of variables of interest over time. In the health field, these variables of interest are, for example, physiological quantities such as blood pressure, heartbeat rates and others, recorded during hospital stays. The expansion of the use of wearable technologies such as smart watches and VR sets increases exponentially the stream of generated data and especially time series data. The use of this data in a way that respects individuals and privacy concerns is a major challenge.

The great wealth of information contained in time series is both a strength and a weakness. Indeed, it is a type of data that it very susceptible to re-identification as was shown by Montjoye et al. [6] and Ingale et al. [12]. Whereas individuals can be re-identified by their characteristics in tabular data, time series are even more sensitive because of their characteristics. The number of measurements in the time series as well as the delay between the measurements can be directly identifying information.

In this paper, we present the application of Chronos, an anonymization algorithm focused on time series and biological signals. The objective of the method we present is to create synthetic records based on original time series records. The newly generated data must retain the information from the original ones while ensuring the privacy of the individuals in the data. It is a new anonymization model that is focused on the individuals and whose privacy protection can be proved.

\section{ANONYMIZATION METHODS}

Synthetic time series anonymization algorithms exist and are already in use to facilitate data sharing. These algorithms can rely on two methods: generating synthetic temporal data and generalizing 


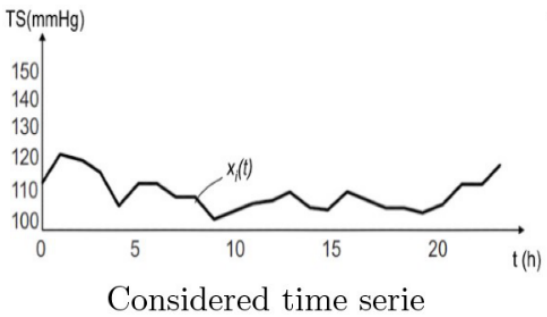

Considered time serie

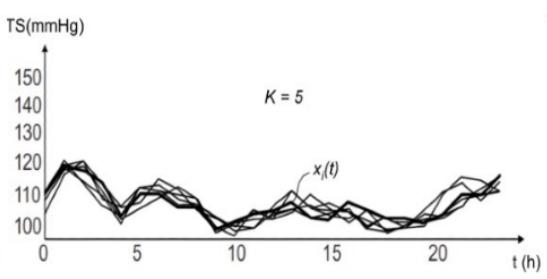

$\mathrm{K}$ closest records

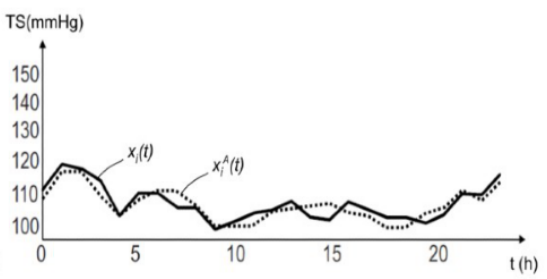

Time serie and its avatar

Figure 1: The avatarisation process

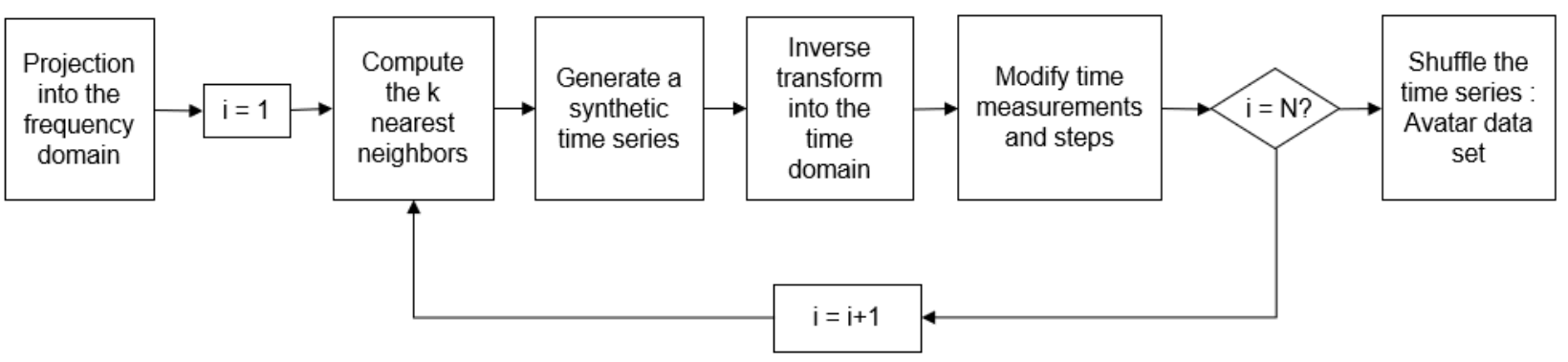

Figure 2: Chronos workflow

or adding noise to the data. The first class of algorithms such as $\mathrm{k}$ anonymity and $(\mathrm{k}, \mathrm{P})$ anonymity by Shou et al [9], are very efficient in keeping the individuals safe, the tradeoff is that they tend to lose valuable parts of the data and can make it hard for it to be used in statistical studies and modeling. The second class of methods rely on neural networks and mostly Generative Adversarial Networks [3] to generate synthetic data that mimics the original data, nevertheless, the privacy preserving capacities of these models must still be proven. The main argument for these methods is plausible deniability as presented by Bindschaedler et al. [1]. Hong et al. [4] presents a review of anonymization techniques for time series data

The synthetic generation methods generally work on time series with similar numbers of measurements and uniformly spaced time steps. In this paper, we present a novel method for generating synthetic time series that takes into account the temporal peculiarities of the data, such as different numbers of measurement and different time steps. It is important to consider these peculiarities, as they can be identifying to the individuals.

\section{INSIGHT ON HOW CHRONOS WORKS}

Chronos is an extension of a method called Avatar [10], Avatar anonymizes tabular data, and uses the closest neighbors to build synthetic new individuals. By analogy, Chronos takes a set of time series each representing an evolution over time of a similar variable, and outputs a set of synthetic time series, called avatars, as an anonymized version of the set of time series. The K nearest neighbors' time series are identified using a predetermined distance computation law, providing distances in the frequency domain between the time series. For each time series of the data set, a synthetic time series is generated from a combination in the frequency domain of the K identified neighbors.

In a second step, and to strengthen the privacy preservation characteristic, the method focuses on the time aspect of the data, by modifying the number of measurement and/or measurement steps and start times. This modification considers the $\mathrm{k}$ neighbors and uses a particular selection process including some randomness.

The Figures 1 and 2 present insights on the method followed for the anonymization of a time series data set.

\section{Class distribution in the training sets}

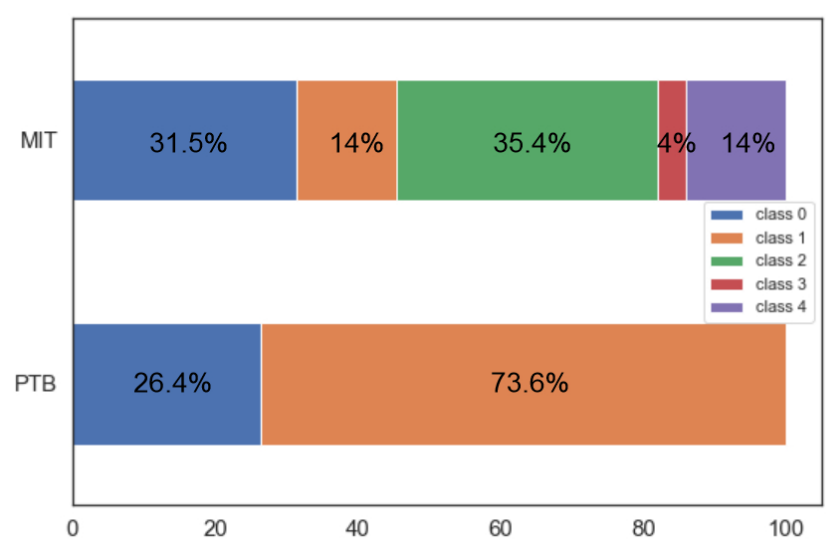

Figure 3: Modalities proportions in the training set 

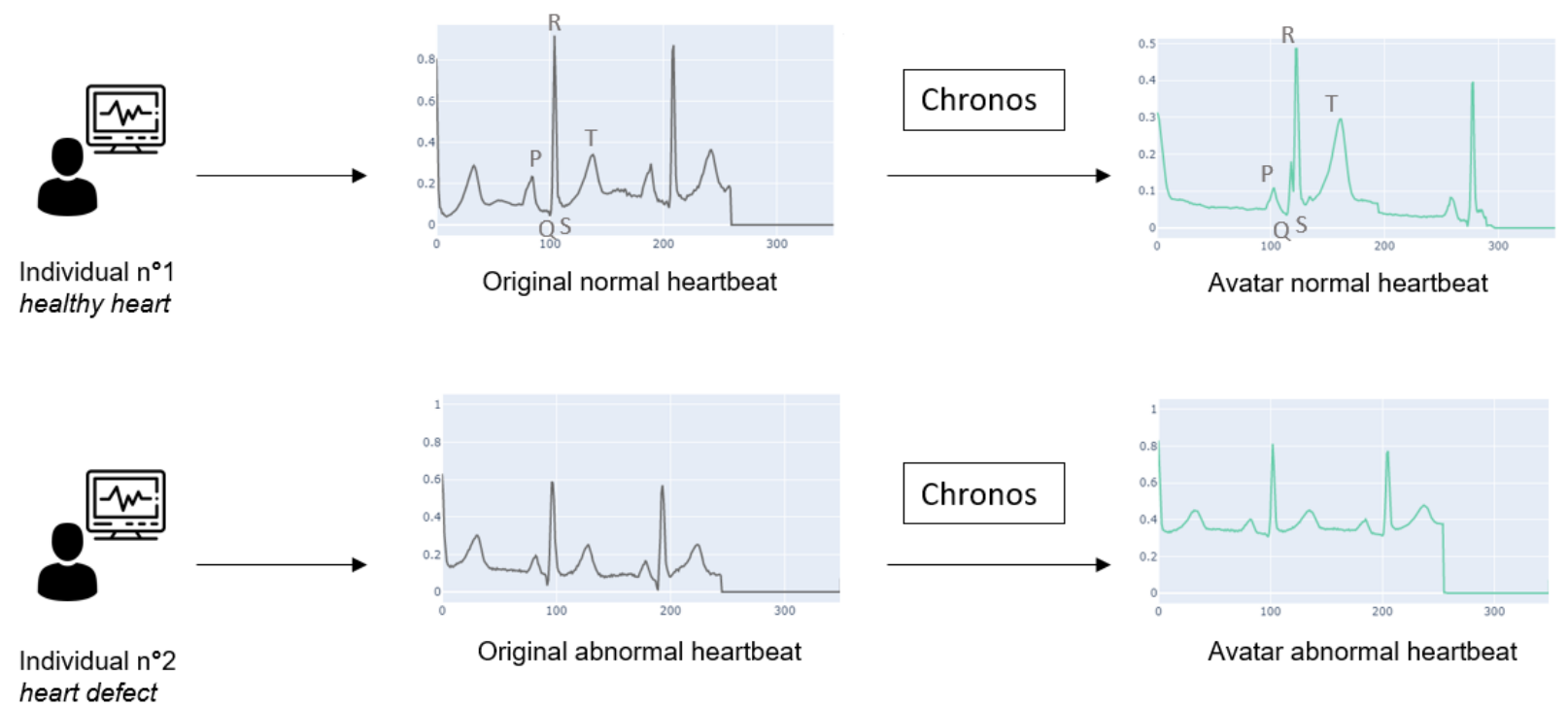

Figure 4: Individual samples from the PTB data set: original vs avatar

\section{APPLICATION TO ELECTROCARDIOGRAM DATA}

To evaluate the efficiency of Chronos, two properties must be checked: how well are the individuals in the data set protected and how well is the signal retained in the anonymized data.

For the first segment, two metrics will be applied to measure the privacy protection provided by the avatars, these metrics are computed using distances between the original data and the avatar records in the frequency domain. For the second segment, the original data will be divided into a training and a testing set, the training set will be anonymized using the Chronos algorithm. An exploratory and descriptive analysis will be done on the original and avatar sets to confirm that they have statistical similarities. Two similar machine learning models will then be trained, the first on the original training data and the second on the anonymized data set, once these models are trained, they will both be tested on the original testing set. If the models have similar accuracy scores and confusion matrices, the anonymization is deemed to retain the signal efficiently.

While Chronos works for any type of time series, it is geared towards health professionals and researchers in the field. We will showcase its efficiency on biological signals, in particular Electrocardiogram time series. We will use two classic ECG data sets, the MIT-BIH Arrhytmia Data Base [7] and the Physikalisch Technische Bundesanstalt (PTB) Diagnostic ECG [2].

The MIT-BIH data set contains 109446 electrocardiogram records, divided into 5 categories following the diagnostic of the patients. The PTB data set contains 14552 records, divided into two categories: normal and abnormal. All of the electrocardiogram records are preprocessed, padded and segmented so that each record corresponds to a single heartbeat. The sampling frequency is $125 \mathrm{~Hz}$ and each record has a length of 188 . Smaller subsets of these data sets will be avatarized, to decrease the imbalance between the classes.
Training subsets of sizes 18630 and 11550 respectively are built from the MIT-BIH and PTB data sets. The Figure 3 below presents the count plots for each class in the training sets.

\subsection{Privacy protection}

To assess the privacy protection of the method, two metrics were developed: Hidden rate and Local cloaking. They are built based on a criterion identified by the article 29 of EDPB, namely, individualization. During the anonymization process, a temporary link between each original time series record and the avatar it created is generated. This link, which is permanently deleted afterwards, can be used to evaluate different attack scenarios.

The individualization criterion means that it must not be possible to identify a particular individual in a data set, and so, the Hidden rate metric measures the probability of making a mistake when an attacker associates an individual with his most similar avatar. It works by projecting the original record and all of the avatars in the frequency domain and computing the proportion of records whose closest avatar was not the one it generated. The hidden rate for the MIT-BIH data set is $84 \%$, for the PTB data set it is $87 \%$.

The Local Cloaking works by measuring the density of each record's surrounding, practically, it computes the median number of avatars between each original record and the avatar it produced. For the MIT-BIH data set, the local cloaking is 5 meaning that each time an attacker tries to identify an individual, he will not be able to differentiate his avatar from 5 other avatars. For the PTB data set, the local cloaking median is 4 .

\subsection{Signal retention}

4.2.1 Visual comparison on the PTB data set. Heartbeat samples are all characterized by their PQRST sequence, which represents one cardiac cycle. The rhythm and amplitude range of these waves is important in the diagnosis of the patients, so it is crucial that 

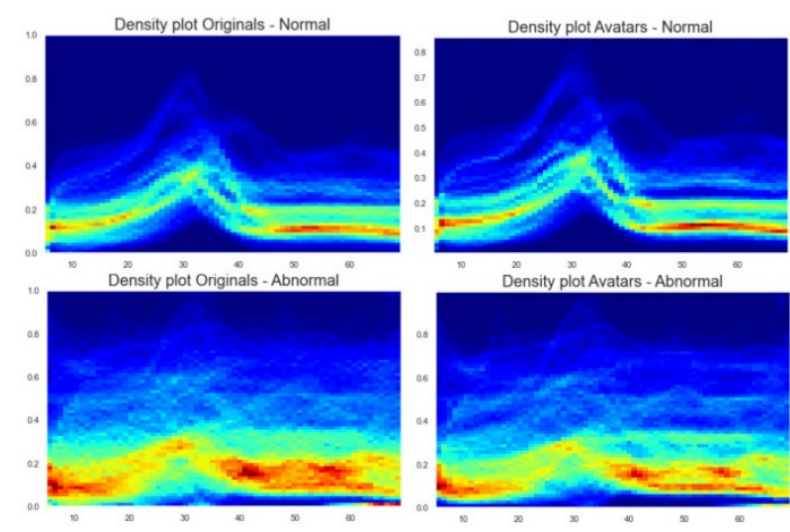

Figure 5: Density of the samples

Chronos retains the shape of this sequence through the anonymization process. There is a visual difference between the heartbeat samples in the normal and abnormal classes in the PTB data set, the PQRST waves are all clearly defined in the normal class, while being harder to differentiate in the abnormal class. This is why we choose this data set to visually compare the original data and the avatars. The first normal and abnormal data points versus their avatar counterparts can be seen in the Figure 4

The density plots in Figure 5 provide more in-depth information on how the normal and abnormal samples are distributed along the time period. The normal samples follow a standard bell shape and peak between the features 20 and 40 , the abnormalsamples do not have a fixed pattern and are widely distributed along the values $[0,0.4]$ in the $y$ axis. The avatars show the same graphical results.

\subsubsection{Prediction models. The PTB data set}

A testing subset of 1000 normal and 2000 abnormal samples is built from the PTB data set to imitate the imbalance of the classes in the training set. After anonymizing the training set, two Extreme Gradient Boosting (XGBoost) algorithms are trained, one on the training set and one on the avatars. The XGBoost model is used in this case because it is fast, does not need a lot of parameters tuning and gives good prediction results in this data set.
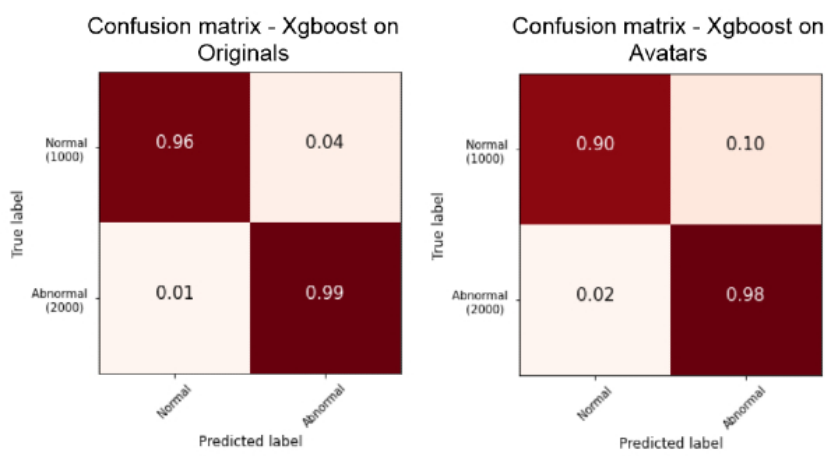

Figure 6: Confusion matrices - XGBoost
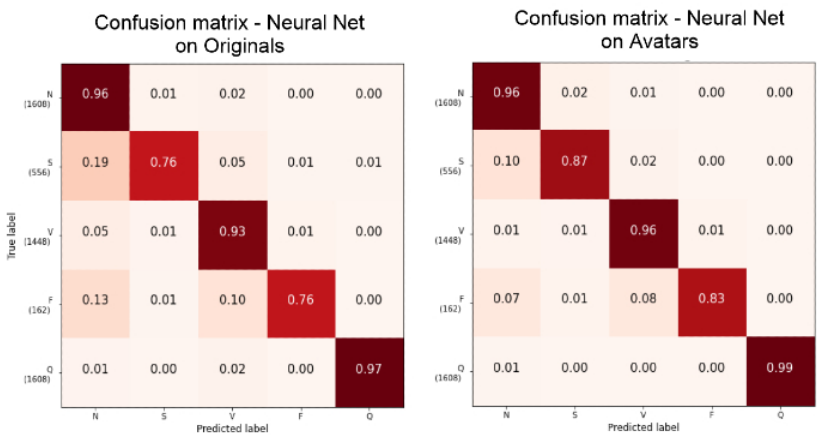

Figure 7: Confusion matrices - Neural Nets

XGBoost yields an accuracy score of $95 \%$ for the anonymized data and an accuracy score of $98 \%$ for the model trained on the original data as can be seen in the Figure 6. The two models are tested on the same data for an objective comparison.

MIT-BIH

The training set is a subset of the MIT-BIH data set comprised of 18652 ECG samples with a similar class distribution as the complete set. The test set has 5382 elements with a similar class distribution as well. For this data set, and due to the multiple classes to predict and the strong class imbalance in the data, a neural network is used to achieve this task. The neural network used here was presented by Kachuee et al. [5], it is build using $1 \mathrm{dConvolution}$ and ReLu activation blocks and performs very well on the original data. The neural network in the Figure 7 has an accuracy score of $95 \%$ when trained on the original data and an accuracy of $92 \%$ on the synthetic data.

\section{CONCLUSION}

In this paper we presented a novel data anonymization method focused on time series data, this method outputs data that is considered anonymous by the GDPR principles and secure for its intended use. The privacy metric ensures that it is formally impossible to re-identify an individual in this data set and thus proves the protection of the sensitive data. The graphical observations and machine learning models prove that the signal is retained through the anonymization process and that the data can still be used for statistical studies, predictions, and classification tasks, demonstrating that we can conciliate data sharing along with the absolute respect of the privacy of the individuals.

While the method we present is rooted in empirical proofs to guarantee the protection and anonymity of the individuals, it is important to remember that for all the anonymization methods, there is always a re-identification risk. This risk is linked to the advances of science in the field and the progression of attacks by malicious entities.

Nevertheless, anonymization can enable a more open data world and help the advances of science by making research data available to all. Data anonymization should become a necessary step in any data processing workflow, making personal information no longer an obstacle to free data sharing without any compromise on the privacy. 


\section{ACKNOWLEDGMENTS}

We would like to thank Emirates NBD and DEWA Research and Development Center for supporting our research.

\section{CONFLICT OF INTEREST}

PA Gourraud is the founder (2008) (www.methodomics.com) and the co-founder of Wedata (2018) (www.wedata.science). He is consulting for major pharmaceuticals companies all dealt with through academic pipelines ((AstraZeneca, Biogen, Boston Scientific, Cook, Edimark, Ellipses, Elsevier, Methodomics, Merck, Mérieux, SanofiGenzyme, WeData). PA Gourraud is board member at AXA mutual insurance company (2021). He has no prescription activity neither drugs nor devices.

\section{REFERENCES}

[1] Vincent Bindschaedler, Reza Shokri, and Carl A. Gunter. 2017. Plausible Deniability for Privacy-Preserving Data Synthesis. Proc. VLDB Endow. 10, 5 (Jan. 2017), 481-492. https://doi.org/10.14778/3055540.3055542

[2] D. Kreiseler, Bousseljot, R. and A. Schnabel. 1995. Nutzung der EKGSignaldatenbank CARDIODAT der PTB über das Internet. Biomedizinische Technik / Biomedical Engineering 40, s1 (1995), 317-318.

[3] Debapriya Hazra, and Yung-Cheol Byun. 2020. SynSigGAN: Generative Adversarial Networks for Synthetic Biomedical Signal Generation. Biology 9, 12 (2020). https://doi.org/10.3390/biology9120441

[4] S. Hong, K. Gurjar, H. Kim, and Yang-Sae Moon. 2013. A Survey on Privacy Preserving Time-Series Data Mining

[5] Mohammad Kachuee, Shayan Fazeli, and Majid Sarrafzadeh. 2018. ECG Heartbeat Classification: A Deep Transferable Representation. 2018 IEEE International Conference on Healthcare Informatics (ICHI) (Jun 2018). https://doi.org/10.1109/ ichi.2018.00092

[6] Yves-Alexandre Montjoye, Cesar Hidalgo, Michel Verleysen, and Vincent Blondel. 2013. Unique in the Crowd: The Privacy Bounds of Human Mobility. Scientific reports 3 (03 2013), 1376. https://doi.org/10.1038/srep01376

[7] G.B. Moody, and R.G. Mark. 2001. The impact of the MIT-BIH Arrhythmia Database. IEEE Engineering in Medicine and Biology Magazine 20, 3 (2001), 45-50. https://doi.org/10.1109/51.932724

[8] Data Protection Working Party. 2014. Opinion on Anonymization Techniques. https://gdprteam.gr/wp-content/uploads/2020/04/ Opinion-052014-onAnonymisation-Techniques-WP216-10.4.2014.pdf

[9] Lidan Shou, Xuan Shang, Ke Chen, Gang Chen, and Chao Zhang. 2013. Supporting Pattern-Preserving Anonymization for Time-Series Data. IEEE Transactions on Knowledge and Data Engineering 25, 4 (2013), 877-892. https://doi.org/10.1109/ TKDE.2011.249

[10] Olivier Breillacq, Yohann Nedelec. 2019. Procédé de création d'avatars pour protéger des données sensibles. https://patents.google.com/patent/FR3091602A1/ fr

[11] Zilong Zhao, Aditya Kunar, Hiek Van der Scheer, Robert Birke, and Lydia Y. Chen. 2021. CTAB-GAN: Effective Table Data Synthesizing. arXiv:cs.LG/2102.08369

[12] M. Ingale, R. Cordeiro, S. Thentu, Y. Park, and N. Karimian. 2020. "ECG Biometric Authentication: A Comparative Analysis," in IEEE Access, vol. 8, pp. 117853117866, 2020, doi: 10.1109/ACCESS.2020.3004464. 